\section{No place like exome}

\section{By Lauren Martz, Staff Writer}

University of Washington researchers haven't wasted any time putting to work their approach to identify rare disease-associated genes based on exome sequencing. ${ }^{1}$ On the heels of an August paper detailing the technique, the team now has used exome sequencing to identify the causal gene for Miller syndrome, a rare Mendelian disorder. The new results suggest that the strategy can be more sensitive than other gene discovery approaches and potentially useful for identifying genes underlying complex diseases. ${ }^{2}$

The exome is the protein-coding region of the genome, which makes up about $1 \%$ of total genetic material and is believed to hold most of the genetic variability. Sequencing the variation-rich exome therefore should reduce the cost and time of mapping genes involved in diseases. ${ }^{3}$

In their previous paper, Jay Shendure and colleagues presented exome sequencing and used it to identify the causal gene for Freeman-Sheldon syndrome, a disease characterized by joint contractures, muscle weakness and fibrosis. That gene was already known, and the results served as proof of concept for the method.

Shendure is assistant professor of genome sciences at the University of Washington.

Now, Shendure and Michael Bamshad, professor of pediatrics and adjunct professor of genome sciences at the university, have applied exome sequencing to Miller syndrome-a disease with a previously unknown causal gene.

Miller syndrome is very rare and involves facial abnormalities, including cleft lip and an undersized lower jaw, as well as incomplete limb development and the absence or webbing of fingers or toes.

In a paper in Nature Genetics, the group sequenced the exomes of four Miller syndrome patients: a pair of siblings and two unrelated individuals.

The group compared the exome sequences of the patients with eight previously sequenced, unaffected individuals and found a mutation in a single candidate gene, dihydroorotate dehydrogenase $(D H O D H)$, that was significantly associated with the disease.

Sanger sequencing of individuals from three additional unrelated families detected the presence of $\mathrm{DHODH}$ mutations in Miller syndrome patients, thus confirming the association.

Shendure told SciBX that previous mapping attempts, such as linkage mapping, had failed to identify causal genes for the syndrome. One reason could be that other analytical methods may exclude genes deemed, in effect, too boring to be involved in disease.

Dennis Ballinger, VP of genomics at Complete Genomics Inc., said a general limitation of candidate gene approaches, which directly test the effect of genetic variants on phenotype, is that they do not target genes like $\mathrm{DHODH}$, which is involved in nucleotide metabolism and considered a housekeeping gene.

"Exome sequencing is more effective because it does not depend on linkage but is nonetheless a genomewide strategy" assuming that causal variants are primarily found in coding sequences, said Shendure.

Radoje Drmanac, cofounder and CSO of Complete Genomics, said previous sequencing methods “either didn't target this gene or could not produce a complete or accurate sequence due to cost or technical limitations. With the identification of the Miller syndrome gene, diagnostic tests can now be developed to screen potential carriers with a view to preventing the transmission of the disease. The development of diagnostics is relatively straightforward when the causal gene is identified."

He added, "The exome approach described targeted most of the coding regions of genes and generated 30 times the coverage with a method that was more affordable than whole-genome sequencing on advanced sequencing platforms."

Complete Genomics is a genotyping services company.

\section{Getting methodical}

Shendure told SciBX that Miller syndrome affects only 30 people in the world and his team therefore has no plans to move forward with DHODH as either a therapeutic target or diagnostic marker.

Instead, his team is now working to improve the exome sequencing approach in two main areas. The first is the capture method, which involves selection of the exome genetic material for sequencing. Second, the group wants to scale up the sequencing and analysis platform to handle a larger number of samples.

He said both types of improvements are necessary to apply the strategy to more complex diseases. For example, using exome sequencing in heterogeneous diseases will require sequencing of a larger number of individuals and new statistical and analytical tools.

"Exome sequencing can be improved to include all exons of all genes by better targeting strategies, and the coverage differences between different exons could be improved by better selection conditions," said Ballinger. "An even lower cost and larger-scale platform will allow for the study of thousands of patients, which would enable the investigation of more common diseases."

Bud Mishra, professor of computer science and mathematics at New York University, said it will be important to improve the strategy's analytical elements. He said a good null model and method of calculating $p$ values will be required to successfully identify disease-associated genes in the future.

The gene for Miller syndrome has not been patented and is unavailable for licensing, according to Shendure.

Martz, L. SciBX 2(47); doi:10.1038/scibx.2009.1720

Published online Dec. 10, 2009 


\section{REFERENCES}

1. Ng, S. et al. Nature 461, 272-276 (2009)

2. Ng, S. et al. Nat. Genet.; published online Nov. 13, 2009; doi:10.1038/ng.499

Contact: Michael Bamshad, University of Washington, Seattle, Wash. e-mail: mbamshad@u.washington.edu

Contact: Jay Shendure, same affiliation as above e-mail: shendure@u.washington.edu

3. Martz, L. SciBX 2(36); doi:10.1038/scibx.2009.1364

COMPANIES AND INSTITUTIONS MENTIONED

Complete Genomics Inc., Mountain View, Calif.

New York University, New York, N.Y.

University of Washington, Seattle, Wash. 